\title{
Modeling the Resolution and Sensitivity of FAIMS Analyses
}

\author{
Alexandre A. Shvartsburg, Keqi Tang, and Richard D. Smith \\ Environmental Molecular Sciences Laboratory, Pacific Northwest National Laboratory, Richland, \\ Washington, USA
}

\begin{abstract}
Field asymmetric waveform ion mobility spectrometry (FAIMS) is rapidly gaining acceptance as a robust, versatile tool for post-ionization separations prior to mass-spectrometric analyses. The separation is based on differences between ion mobilities at high and low electric fields, and proceeds at atmospheric pressure. Two major advantages of FAIMS over condensedphase separations are its high speed and an ion focusing effect that often improves sensitivity. While selected aspects of FAIMS performance are understood empirically, no physical model rationalizing the resolving power and sensitivity of the method and revealing their dependence on instrumental variables has existed. Here we present a first-principles computational treatment capable of simulating the FAIMS analyzer for virtually any geometry (including the known cylindrical and planar designs) and arbitrary operational parameters. The approach involves propagating an ensemble of ion trajectories through the device in real time under the influence of applied asymmetric potential, diffusional motion incorporating the high-field and anisotropic phenomena, and mutual Coulomb repulsion of ionic charges. Calculations for both resolution and sensitivity are validated by excellent agreement with measurements in different FAIMS modes for ions representing diverse types and analyte classes. (J Am Soc Mass Spectrom 2004, 15, 1487-1498) @ 2004 American Society for Mass Spectrometry
\end{abstract}

$\mathrm{A}$ major endeavor of modern analytical chemistry is building the tools to address samples of ever-increasing complexity. This is primarily driven by the need to characterize biological analytes, which have become so topical as to create new scientific fields, e.g., proteomics [1] and metabolomics [2]. The principal analytical technology of those fields is mass spectrometry (MS), revolutionized by coupling with ESI and MALDI soft-ionization sources. Even with the formidable power of state-of-the-art MS, nearly all real-world samples are too complex to be handled without prior separations (particularly when lessabundant constituents are of interest). Well-established methods involve separations in a solid (gel techniques such as sodium dodecylsulfate-polyacrylamide gel electrophoresis (SDS-PAGE) and 2-D gel [3]) or liquid (for example, liquid chromatography (LC) [4], strong-cation exchange (SCX) [5], and capillary electrophoresis [6]). By the nature of condensed phase, all separations take a significant time: generally from minutes to hours. More complex analytes often necessitate multidimensional separations such as SCX/reverse phase LC (e.g., the MudPIT protocol [7]), which can last tens of hours. That is far too long for the throughput needs of modern proteomics and metabolomics.

Published online September 11, 2004

Address reprint requests to Dr. R. D. Smith, Biological Systems Analysis and Mass Spectrometry, Pacific Northwest National Laboratory, 3335 Q Avenue (K8-98), P.O. Box 999, Richland, WA 99352, USA.
The search for powerful yet fast separations that would effectively utilize the high speed of MS analyses has logically focused attention on gas-phase methods. While gas chromatography is swift, it is incompatible with large biomolecules as they are not volatile and thus cannot reversibly adsorb on solid phases under equilibrium conditions. This leaves the separations based on ion mobility spectrometry (IMS) [8-16]. In classic IMS, analyte ions are pulled at a constant velocity $(v)$ through a non-reactive buffer gas by a timeindependent, nearly uniform electric field. In the weakfield limit, $v$ is proportional to the field intensity $(E)$, and the $v / E$ ratio is termed mobility $(K)$. Mobility is usually adjusted to the standard buffer gas number density $(N)$, yielding the reduced mobility $\left(K_{0}\right)$. This quantity (at a particular temperature, $T$ ) is a unique property of ion/buffer gas combination. Typically IMS is implemented in drift tubes featuring an axial electric field established by rings at defined voltages stacked along the tube [10-12]. An ion packet containing species with different $K_{0}$ is injected into a drift tube and fractionated while traversing it. If the temporal separation between "eluting" components exceeds the MS analysis timescale, a mass spectrometer joined to the IMS terminus can probe the components one at a time. IMS has now been coupled to quadrupole MS [10], time-of-flight MS [13], and FTICR [14]. The IMS/TOF hybrid that disperses analyte mixtures in mobility and mass dimensions simultaneously is a promising new 
platform for high-throughput analytical applications $[15,16]$.

Another ion mobility-based technique that has emerged lately is FAIMS [17-42]. The FAIMS analysis is based on the fact that ion mobilities depend on the electric field intensity: The $K_{0}$ quantity above is the limit of $K_{0}(E)$ function at $E \rightarrow 0$. Specifically, $K_{0}(E)$ can be expressed $[23,30]$ as a polynomial of even powers of $E / N$ :

$$
K_{0}(E)=K_{0}\left(1+a(E / N)^{2}+b(E / N)^{4}+c(E / N)^{6}+\ldots\right),
$$

Rigorously, eq 1 is an infinite series, but at realistic field intensities the terms of sixth order and higher are insignificant $[23,30]$. When $E$ increases beyond the low-field limit, mobilities may increase (referred to as species of Type A), decrease (Type C), or first increase and then decrease at still higher $E$ (Type B) [21, 22]. Usually, small and structurally rigid ions (including all monatomics) belong to Type A, and large and flexible ions (e.g., proteins and polypeptides) belong to Type $\mathrm{C}$. Both cations and anions may belong to any type. For all types, the effect generally becomes significant only for very strong fields [22], on the order of $10^{4} \mathrm{~V} / \mathrm{cm}$ (at $1 \mathrm{~atm}$ ) which is close to the point of electrical breakdown in gases. This phenomenon can be exploited to separate ions by the difference between mobilities at high fields $\left(K_{\mathrm{h}}\right)$ and low fields. In FAIMS, this is implemented by passing a stream of non-reactive gas that contains analyte ions between a pair of electrodes of a planar or cylindrical shape carrying an asymmetric time-dependent periodic potential $V_{\mathrm{D}}(t)$. Under its influence, ions move towards either electrode and are neutralized, providing the basis for separation [21, 22]. The integral of $V_{\mathrm{D}}(t)$ over each period must be null, but time-averaged positive and negative voltages are not equal. An example is a sum of a sinusoidal waveform and its scaled phase-shifted harmonic [23, 30]:

$$
V_{\mathrm{D}}(t)=[f \sin w t+\sin (h w t-\phi)] V_{\max } /(f+1),
$$

The experimental work so far has adopted eq 2 with the parameters of $h=2, \phi=\pi / 2$, and $f=2,3$, or 4 , with the most data available for $f=2$. While the computational model described herein can treat any combination of these parameters, all actual calculations below use $h=$ $2, \phi=\pi / 2$, and $f=2$. By eq $2, V_{\max }$ equals the maximum instantaneous absolute voltage, termed the "dispersion voltage" (DV). Had $K(E)$ been constant, the displacements of ions during the periods of positive and negative $V_{\mathrm{D}}$ would have fully canceled. Since the mobilities in those periods differ, the displacements do not cancel completely and ions drift towards either electrode, depending on the ion type [21, 22]. A particular ion may be kept in position (i.e., not lost to an electrode) by applying (on top of $V_{\mathrm{D}}$ ) a DC compensation voltage $(\mathrm{CV})$ that would cause a drift exactly opposite to that produced by $V_{\mathrm{D}}(t)$. The $\mathrm{CV}$ required for each ion is set by the difference between its high- and (a)

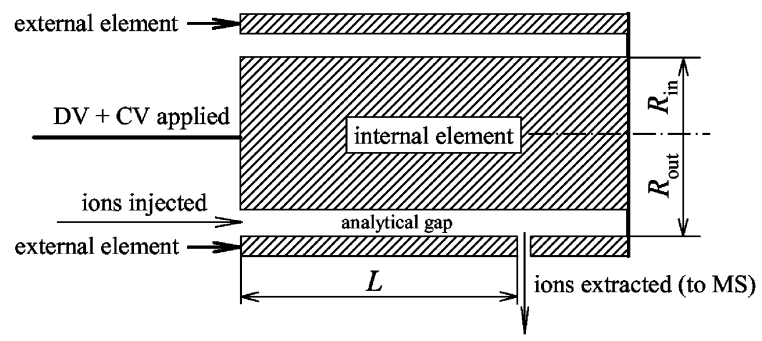

(b)

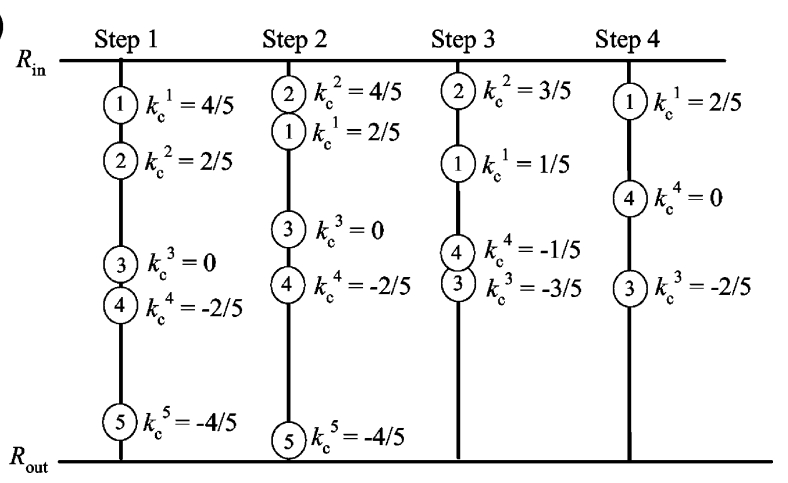

Figure 1. (a) A scheme of cylindrical FAIMS design with relevant dimensions. (b) A diagram illustrating the $k_{\mathrm{c}}^{j}$ factors assigned at four successive simulation steps for an array of $n$ ions with arbitrary radial coordinates in the FAIMS gap. Here $n=5$, the actual simulation typically runs with $n=200-1000$. In step 2, ions 1 and 2 permuted their positions. In step 3 , ions 3 and 4 permuted their positions and ion 5 hits the external element. In step 4, ion 2 hits the internal element.

low-field mobilities governed by eq 1 , and thus is the characteristic property of a species. Hence ideally only one component of ionic mixture may pass through FAIMS at a specific $C V$, and an analyte spectrum may be obtained by scanning the CV [21, 22].

The original FAIMS device featured planar electrodes [17]. The concentric cylinder geometry [20] has subsequently become popular [21-42] because of ion focusing in inhomogeneous electric field [21, 22]. Since this focusing is central to the operation of cylindrical FAIMS and its competition with the planar design [17-19], we now discuss it in detail. The dispersion field between two cylinders (Figure 1a) at a radial coordinate $r$ is:

$$
E_{\mathrm{D}}(r, t)=V_{\mathrm{D}}(t) /\left[r \ln \left(R_{\text {out }} / R_{\text {in }}\right)\right],
$$

where $R_{\text {out }}$ and $R_{\text {in }}$ are respectively the inner radius of the outer cylinder and outer radius of the inner cylinder [22]. Then the field of CV (compensation field) needed to offset the net displacement due to DV is also a function of $r$. For brevity, the following case is for Type A ions, but the argument equally applies to Type $\mathrm{C}$ ions [22]. As a corollary of eq 1 , the compensation field required to achieve equilibrium increases with increasing dispersion field faster than linearly. For example, one design [21,22] features $R_{\text {in }}=0.7 \mathrm{~cm}$ and $R_{\text {out }}=0.9$ $\mathrm{cm}$. At $V_{\mathrm{D}}=V_{\max }=-3.3 \mathrm{kV}$ applied to the internal 
Table 1. Mobility parameters assumed for the ions modeled in this work (listed in the order of appearance in the text)

\begin{tabular}{|c|c|c|c|c|}
\hline System & Reference & $K_{0}(0), \mathrm{cm}^{2} /(\mathrm{Vs})$ & $a, \mathrm{Td}^{-2}$ & $b, \mathrm{Td}^{-4}$ \\
\hline $\mathrm{Cl}^{-}$in air & {$[23]$} & 2.875 & $1.87 \times 10^{-5}$ & $6.44 \times 10^{-9}$ \\
\hline 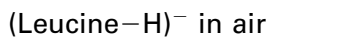 & {$[30]$} & 2.18 & $5.43 \times 10^{-6}$ & $-1.85 \times 10^{-10}$ \\
\hline$(\text { Isoleucine }-\mathrm{H})^{-}$in air & [30] & 2.18 & $5.15 \times 10^{-6}$ & $-5.78 \times 10^{-11}$ \\
\hline $\mathrm{H}^{+}$Tryptophan in air & [30] & 2.09 & $1.27 \times 10^{-6}$ & $1.8 \times 10^{-12}$ \\
\hline $\mathrm{H}^{+}$Glycine in $\mathrm{N}_{2}{ }^{\mathrm{a}}$ & {$[30]$} & 2.32 & $9.65 \times 10^{-6}$ & $-4.31 \times 10^{-10}$ \\
\hline $\mathrm{HSO}_{4}^{-}$in air $^{\mathrm{b}}$ & {$[25]$} & 2.50 & $1.66 \times 10^{-5}$ & $-4.95 \times 10^{-10}$ \\
\hline $\begin{array}{l}\left(\mathrm{H}^{+}\right)_{12}^{4} \text { Bovine Ubiquitin } \\
\quad \text { (major conformer) in } \mathrm{N}_{2}\end{array}$ & {$[40]$} & 1.30 & $-2.34 \times 10^{-6}$ & $-1.6 \times 10^{-12}$ \\
\hline $\begin{array}{l}\left(\mathrm{H}^{+}\right)_{12} \text { Bovine Ubiquitin } \\
\text { (minor conformer) in } \mathrm{N}_{2}\end{array}$ & [40] & 1.30 & $-1.99 \times 10^{-6}$ & $-1.20 \times 10^{-11}$ \\
\hline
\end{tabular}

${ }^{\mathrm{a}}$ The value for $b$ differs from that listed in reference [30]. The adjustment was needed to obtain the measured [29] $\mathrm{CV}=-9.85 \mathrm{~V}$ (at $\mathrm{DV}=3 \mathrm{kV}$ ) instead of $\mathrm{CV}=-11.0 \mathrm{~V}$ calculated using [30] $b=-1.52 \times 10^{-10} \mathrm{Td}^{-4}$. This discrepancy may derive from that the values in reference [30] are for air, whereas the measurements presented here are for $\mathrm{N}_{2}$. The $K(E)$ trends in $\mathrm{O}_{2}$ and $\mathrm{N}_{2}$ may differ significantly [62].

${ }^{\mathrm{b}} K_{0}(0)$ was estimated and $a, b$ were derived from measured CVs.

${ }^{\mathrm{c}} K_{0}(0)$ is from references $[40,42,63]$ and $a, b$ were derived from measured CVs.

electrode, the values of $E$ inside the device range from $\sim-18,760 \mathrm{~V} / \mathrm{cm}$ for $r=R_{\text {in }}$ to $\sim-14,590 \mathrm{~V} / \mathrm{cm}$ for $r=$ $R_{\text {out }}$, with $\sim-16,410 \mathrm{~V} / \mathrm{cm}$ at the midpoint $(r=0.8 \mathrm{~cm})$. For a $\mathrm{Cl}^{-}$ion [23], the equilibrium compensation field should range from $557 \mathrm{~V} / \mathrm{cm}$ to $216 \mathrm{~V} / \mathrm{cm}$, with 338 $\mathrm{V} / \mathrm{cm}$ at the midpoint (the mobility parameters for all ions discussed in this paper are listed in Table 1). However, the $\mathrm{CV}=67.9 \mathrm{~V}$ that produces the correct 338 $\mathrm{V} / \mathrm{cm}$ at $r=0.8 \mathrm{~cm}$ would yield only $386 \mathrm{~V} / \mathrm{cm}$ at $r=$ $R_{\text {in }}$ but $300 \mathrm{~V} / \mathrm{cm}$ at $r=R_{\text {out }}$. Calculations along these lines show that actual compensation fields are below their equilibrium values for all $r<0.8 \mathrm{~cm}$ and above them for $r>0.8 \mathrm{~cm}$. Hence, a $\mathrm{Cl}^{-}$ion would experience a net outward force when $r<0.8 \mathrm{~cm}$ and inward force when $r>0.8 \mathrm{~cm}$. In other words, an inhomogeneous electric field creates a pseudopotential well that seeks to confine $\mathrm{Cl}^{-}$to its equilibrium radius $\left(R_{\mathrm{eq}}\right)$. That radius does not have to equal $\left(R_{\text {in }}+R_{\text {out }}\right) / 2$ : focusing can be achieved at any $R_{\text {in }}<r<R_{\text {out }}$ by an appropriate choice of CV.

The advantages and disadvantages of FAIMS in comparison with regular IMS become apparent from a parallel with mass spectrometry, where those are analogous to a quadrupole MS and TOF MS, respectively. The key advantage of FAIMS is that it is a continuous technique that is readily compatible with continuous ion sources such as ESI, whereas IMS is a pulsed technique that cannot utilize continuous sources without a means for ion accumulation between pulses or a drastic signal loss. The major shortcoming of FAIMS is that it is a scanning technique that inherently involves a trade-off between resolution and sensitivity due to the duty cycle, which is not necessarily the case for dispersion techniques like IMS.

The two principal performance measures of any analytical technique are resolution and sensitivity. For IMS/MS hybrid, the IMS sensitivity means its transmission coefficient to the MS stage. The factors governing resolution and transmission for regular IMS are wellestablished in theory and experiment. For instance, the IMS resolving power is (assuming a negligible initial ion pulse width) proportional to $(E L / T)^{1 / 2}$, where $L$ is the ion path length [43]. A grasp of the dependence of IMS performance on instrumental parameters has successfully guided the development of that technology, in particular allowing its resolving power to be raised by an order of magnitude through the use of higher fields, longer tubes, and lower temperatures [11, 44]. Knowledge of achievable resolution and sensitivity is helpful in both experimental design and data interpretation. For example, it has shown that certain peaks observed in IMS are unreasonably broad and thus represent two or more different species, even when the peak shape revealed no abnormalities [45]. Finally, having a clear expectation for performance in any regime permits an immediate recognition and diagnosis of instrumental malfunctions.

No similar insight presently exists for FAIMS: The factors governing the peak width and transmission have remained obscure. The number of experimental variables in FAIMS is greater than that in IMS: in addition to the field intensity, ion path length, and gas properties (composition, temperature, and pressure), one may also adjust the shape and width of the gap between electrodes (analytical gap), the profile and frequency of asymmetric waveform, the CV scan speed, and the ion residence time in the device (determined by the gas flow speed through the gap). This flexibility (and complexity) suggests that the performance might be materially enhanced if the interplay of all controlling instrumental parameters was understood as well as for IMS. In particular, the resolving power of current FAIMS for most analytes of interest is only $\sim 10$, which is much in need of improvement [28]. While the ion trajectory simulations of Guevremont and co-workers $[22,34]$ have been valuable for understanding the mechanism of ion focusing and trapping in FAIMS devices, the actual waveform (eq 2) was approximated by a rectangular potential and the diffusion and Coulomb repulsion were neglected.

Here we report a computational capability for simulating the behavior of ions in FAIMS devices using 
classical molecular dynamics. This software can handle species with arbitrary $K(E)$ dependence in both planar and cylindrical designs with any geometric and operational parameters. The underlying physical model incorporates the diffusion and Coulomb repulsion (the space-charge effect) that has proven extremely important. Application of this model to published data demonstrates an excellent agreement with experiment.

\section{Computational Model}

Our model of FAIMS operation involves propagating an ensemble of classical molecular dynamics trajectories through the analytical gap defined by arbitrary $R_{\text {in }}$ and $R_{\text {out }}$ (Figure 1a). Both cylindrical [20-42] and planar [17-19] geometries can be considered, with the latter viewed as the limiting case of very large $R_{\text {in }}$ and $R_{\text {out }}$. At this time, the model does not address the cylindrical geometry with a hemispherical "endcap" for ion extraction, a feature of recent designs [26, 32, 35, 39]. In our treatment, ions experience three effects: directed drift due to $E_{\mathrm{D}}(r, t)$, random diffusion, and mutual Coulomb repulsion. The directed drift may occur only along the $r$ vector because the electric field between concentric cylinders has no axial component (disregarding edge effects). Both the diffusion and Coulomb repulsion cause ions to move in the other two orthogonal directions along the gap. However, FAIMS is a continuous technique, meaning the ion density along the gap must be constant in time. Therefore both the time-averaged velocity of any ion and the mean velocity of a statistically representative ion ensemble along the gap must equal the gas flow velocity. So the simulation evolves in one dimension $(r)$, with the coordinate frame moving along the gap at the gas flow velocity. This, of course, is an approximation since a random motion of individual ions along the gap may still affect the result. In particular, this motion would create a distribution of ion residence times in FAIMS, rather than a single residence time $\left(t_{\text {res }}\right)$ assumed herein.

Our model represents the ion population by a number $(n)$ of identical "macroions" (below simply "ions") disposed along $r$. The electric field created by all $n$ ions along $r$ is set to match the field of the actual surface charge density in FAIMS. That density is determined from the device geometry, ion current $(I)$, and $R_{\text {eq }}$ (depending on the specific ion and operational conditions) as $\sigma=I t_{\text {res }} /\left(2 \pi R_{\mathrm{eq}} L\right)$, where $L$ is the ion path length. The field intensity is evaluated in the quasiplanar approximation as $E_{\sigma}=\sigma /\left(2 \varepsilon_{\mathrm{o}}\right)$, where $\varepsilon$ is the dielectric constant of gas and $\varepsilon_{\mathrm{o}}$ is the permittivity of vacuum. This approximation should be reasonable as long as $\left(R_{\text {out }}-R_{\text {in }}\right) \ll R_{\text {eq }}$, which holds in almost all FAIMS designs. Since all gases at atmospheric pressure have $\varepsilon<1.01\left(\varepsilon=1.0005\right.$ for $\mathrm{N}_{2}$ and $\left.\mathrm{O}_{2}\right)$, we use $\varepsilon=1$. The electric field of Coulomb repulsion acting on each ion is then derived as $E_{\sigma}$ for the initial current at the start of simulation $\left(I_{\mathrm{in}}\right)$ scaled by $k_{\mathrm{c}}-$ the difference between the fractions of other ions located on its two sides relative to the original $n$. This process is illustrated in Figure $1 \mathrm{~b}$. Note that the values of $k_{\mathrm{c}}$ for all ions differ at any moment in time and may be positive or negative (ranging between -1 and 1). That is, the space charge is equally likely to push an ion towards either FAIMS electrode. The $k_{\mathrm{c}}$ for each ion is continually updated, depending on the relative positions of other ions. The final formula for the electric field experienced by any $j$ th ion ( $j$ ranging from 1 to $n$ ) reads:

$$
\begin{aligned}
& E_{\text {tot }}^{j}(r, t)=\{\mathrm{DV}[2 \sin w t+\sin (2 w t \\
&-\pi / 2)] / 3+\mathrm{CV}\} /\left[\operatorname{r} 1 \mathrm{n}\left(R_{\text {in }} / R_{\text {out }}\right)\right]+k_{c}^{j}(t) I_{\text {in }} t_{\text {res }} /\left(4 \pi R_{\text {eq }} L \varepsilon_{\mathrm{o}}\right),
\end{aligned}
$$

If an ion hits an electrode (the condition $r_{\mathrm{j}}>R_{\text {out }}$ or $r_{\mathrm{j}} \leq$ $R_{\text {in }}$ exists), it is removed from the simulation and $k_{\mathrm{c}}^{\mathrm{j}}$ values for all other $j$ are adjusted accordingly. Thus, the current and the space-charge effect properly attenuate when ions are lost to FAIMS filtering action.

The starting ion coordinates $r^{j}$ may be set according to any desired distribution. We have tested three cases: ions are injected at the gap midpoint $\left[r^{\mathrm{j}}=\left(R_{\text {in }}+\right.\right.$ $R_{\text {out }}$ )/2], at their equilibrium radius $r^{\mathrm{j}}=R_{\mathrm{eq}}$, or distributed evenly and symmetrically over an adjustable distance $l$ across the gap $\left[r^{j}=\left(R_{\text {in }}+R_{\text {out }}-l\right) / 2+j l / n\right]$. Other options (e.g., a Gaussian or a random distribution) may be handled just as readily. The initial conditions (within reason) had only a small influence on the outcome of simulations for cylindrical FAIMS presented here, because the focusing moves all ions into a band around $R_{\text {eq }}$ early in the process, thus rapidly erasing the memory of original distribution. This would not necessarily apply for poor ion focusing conditions, where $K(E) \sim K_{0}(0)$, or absolute $K(E)$ is small, and/or FAIMS geometry has no or low curvature: in general, the initial ion coordinates should be chosen judiciously. All simulations below use $r^{\mathrm{j}}=\left(R_{\text {in }}+R_{\text {out }}\right) / 2$.

In each simulation step lasting $\Delta t$, a $j$ th ion is first moved by the displacement caused by total electric field:

$$
\Delta r_{\mathrm{E}}^{j}(r, t)=v^{j}(r, t) \Delta t=K\left(E_{\text {tot }}^{j}(r, t)\right) E_{\text {tot }}^{j}(r, t) \Delta t,
$$

where $K(E)$ is given by series (eq 1) truncated at the second term. Then the ion is further moved due to diffusion. That displacement $\left(\Delta r_{D}^{j}\right)$ is randomly selected (separately for each $j$ ) with the probability given by the Gaussian distribution

$$
p\left(\Delta r_{\mathrm{D}}^{j}\right)=\left(4 \pi D_{L} \Delta t\right)^{-1 / 2} \exp \left[-\left(\Delta r_{\mathrm{D}}^{j}\right)^{2} /\left(4 D_{L} \Delta \mathrm{t}\right)\right]
$$

Here $D_{\mathrm{L}}$ is the longitudinal diffusion constant that regulates the diffusion of ions drifting in gases along the drift direction. In the low-field limit, $D_{\mathrm{L}}$ is related to mobility by the Einstein equation $\left(D=k_{\mathrm{B}} T K / q\right)$, where $k_{\mathrm{B}}$ is the Boltzmann constant and $q$ is the ionic charge [46, 47]. At high fields, the relationship gets quite 
complex. The diffusion becomes anisotropic, i.e., the constant becomes direction-specific [46]. The above equation (for $D_{\mathrm{L}}$ ) generalizes to $[46,47]$ :

$$
D_{L}=k_{B} \mathrm{~T}_{\mathrm{L}} \mathrm{K}(E)[1+d \ln K / d \ln (E / N)] / q,
$$

where $T_{\mathrm{L}}$ is the "longitudinal temperature" (the measure of ions random energy along the drift axis). The key problem is the evaluation of $T_{\mathrm{L}}$ that depends not only on $E$ and relative masses of the ion $(m)$ and buffer gas molecule $(M)$, but also on the detailed physics of ion-neutral collisions that manages the deposition of kinetic energy into colliding particles and its partition between their translational degrees of freedom. The relevant quantities (e.g., the angular behavior of the differential cross-section) are determined by the two-body interaction potential, and thus depend on the identity of ion and gas molecule $[46,47]$. To create a universal model, we have to adopt some approximation for $T_{\mathrm{L}}$, particularly as different derivations yield slightly different results $[46,47]$. In this work we use the expression:

$$
T_{\mathrm{L}}=T+[1+m /(m+0.5 M)] M v^{2}(r, t) /\left(3 k_{\mathrm{B}}\right)
$$

following from the derivation for isotropic scattering [46]. We highlight that $T_{\mathrm{L}}$ (and thus $D_{\mathrm{L}}$ ) are timedependent through the variation of $v(r, t)$ : ions diffuse more during the high-field portion of the FAIMS cycle. Other more sophisticated formalisms may be more accurate [47], especially for extreme fields exceeding $100 \mathrm{Td}$. For the moderately high fields of 30-100 Td in present FAIMS technology, eq 8 should produce reasonable results. Notably, the coefficient with the second term in eq 8 ranges from 1 to 2 . For light ions (such as $\mathrm{Na}^{+}$and $\mathrm{Cl}^{-}$), a typical value would be $1.5-1.7$. For biomolecular ions, one normally finds $m \gg M$, and the value is 1.9-2.0. A similar expression in the twotemperature treatment of IMS mobility theory [48] has a coefficient of 1 . This difference is due to our concern with diffusion along the drift direction, which is faster than the orientationally-averaged quantity.

The displacement due to diffusion found from eqs 6-8 completes a simulation step. At this point, we check for ions that have hit an electrode and eliminate them (if any) from the simulation. The next step starts from reevaluating eq 4 for new $r^{j}$ of all ions, except that a laborious update of $k_{\mathrm{c}}$ array is performed once per FAIMS cycle. The $\Delta t$ value is selected to ensure an integer number of steps in the FAIMS cycle, so a cycle always starts from a whole step. We use 200 steps per cycle, which always guarantees convergence. The simulation runs for the full residence time, estimated at $0.1-0.5 \mathrm{~s}$ and most typically $\sim 0.2 \mathrm{~s}$ in cylindrical designs [26, 32, 33, 35]. Unless indicated otherwise, we adopt $t_{\text {res }}=0.2 \mathrm{~s}$. With the waveform frequency $(w / 2 \pi)$ of current cylindrical FAIMS instruments in the $200-750 \mathrm{kHz}$ range, $t_{\text {res }}$ comprises $2 \times 10^{4}-4 \times$
$10^{5}$ cycles, which means $4 \times 10^{6}-8 \times 10^{7}$ steps of 7-24 ns duration each. We start with $n=200-1000$ ions, which provides a good convergence in all cases except when only few ions survive FAIMS, and so the number and properties of survivors may be not representative. In that scenario, a greater $n$ should be used. Since modeling of the space-charge effect requires accounting for all pairwise interactions, part of computation scales as $n^{2}$ and simulations for high $n$ are expensive. The model has been implemented in a Fortran77 code. As a guide, one execution on a 3.2 $\mathrm{GHz}$-processor PC normally takes several minutes to several hours, depending on the input parameters.

A key assumption underlying eq 5 is that the instantaneous ion velocity always corresponds to the instantaneous field; in other words the field varies slowly enough for the velocity to adjust. The characteristic relaxation time [23] is $\tau=2 m K(E) / q$. For small and midsize ions in air, $\mathrm{N}_{2}$, and $\mathrm{O}_{2}$ gases that are common FAIMS buffers, typical values are $0.1-1$ ns, e.g., $0.2 \mathrm{~ns}$ for $\mathrm{Cl}^{-}$and $0.6 \mathrm{~ns}$ for (Leucine $\left.-\mathrm{H}\right)^{-}$[30]. These times are at least an order of magnitude shorter than $\Delta t$ in our simulations, and the relaxation effect should be immaterial. Large macromolecular ions (such as proteins) are much heavier, but have lower mobility. So their relaxation times are still modest, for example $t \sim 4.5 \mathrm{~ns}$ for even the most compact conformation of apomyoglobin $(m=17 \mathrm{kDa})$ found in ESI/IMS/MS experiments [49] $\left\{K_{0}(0) \sim 0.5 \mathrm{~cm}^{2} /(\mathrm{Vs})\right.$ for $(+4)$ charge state, estimated for air from the measured cross-section of $\left.\sim 1500 \mathrm{~A}^{2}\right\}$. Using $\mathrm{He}$ as a major component of FAIMS gas has recently been found of benefit for both resolution and sensitivity [39, 40]. Because He is light, physically small, and has a low polarizability, ion mobilities in He are 3-4 times higher than those in air. For example, the same apomyoglobin ion in He would have $t \sim 13 \mathrm{~ns}$, a value comparable to $\Delta t$ in our simulations. Thus the relaxation might introduce a small correction for heaviest ions with compact conformations (especially lowcharge protein species generated by MALDI [50] rather than ESI), particularly when light gases such as He are major constituents of the FAIMS medium. For most cases, including those considered in this study, this correction is negligible.

We emphasize that the present model is for pure buffer gases only, not the mixtures of two or more gases employed in some experiments. The $D_{\mathrm{L}}$ quantities in mixtures may differ from those predicted by Blanc's law drastically, so eq 7 may miscalculate the diffusion speed [51,52]. Of greater consequence, the mobilities of ions in gas mixtures may also significantly deviate from those predicted by Blanc's law [52,53], so the eq 1 and the basic relationship of $\mathrm{CV}$ to $\mathrm{DV}$ ensuing from it might not apply. Indeed, unexpected results of analyses in gas mixtures (e.g., $\mathrm{CO}_{2}$ and $\mathrm{N}_{2}$ ) have been noted [32]. The development of adequate theory for FAIMS separations in gas mixtures remains a challenge for future work. 


\section{Comparison with FAIMS Experiments}

We now evaluate this computational model using representative FAIMS experimental data, which are customarily presented as CV spectra acquired at a series of DV values. These allow modeling the peak profile of any one spectrum (i.e., the transmitted current, $I_{\text {out }}$ versus $C V$ at a fixed DV) and the sensitivity (i.e., $I_{\text {out }}$ at a peak apex) as a function of DV. In simulation, $I_{\text {out }}$ is determined as $I_{\text {in }}$ multiplied by the fraction of ions that have passed through FAIMS.

\section{Modeling Resolution and Sensitivity-the Test Ground of (Leucine - H)}

Analysis of proteolytic digests that consist mostly of small peptides is central to the modern proteomics procedure, and individual amino acids are frequently encountered in metabolomic analyses [54]. Separation of peptides and amino acids in FAIMS is significantly orthogonal to both IMS and MS dimensions [28, 33, 35]. The leucine/isoleucine mixture has presented a good assessment of FAIMS separation capability [28], and a precise calculation of resolution would be important in designing such an experiment.

(Leucine $-\mathrm{H})^{-}$is an A-type ion that passes FAIMS in N1 mode. Computed peak profiles are compared with the measurement [28] in Figure 2, and representative trajectories are plotted for inspection in Figure 3. The geometric parameters $[21,22,28]$ were $R_{\text {in }}=0.7 \mathrm{~cm}$, $R_{\text {out }}=0.9 \mathrm{~cm}$, and $L=9 \mathrm{~cm}$. If both the diffusion and Coulomb repulsion are ignored, all trajectories are totally deterministic: an ion always either passes FAIMS or not, depending on the DV, CV, and initial coordinate. The calculated peak is a rectangle between the CV values of $6.47 \mathrm{~V}$ and $8.98 \mathrm{~V}$ (Figure 2a), while the measured profile is many times narrower. The addition of diffusion produces features that sharpen towards the top: the probability of any ion hitting an electrode is now finite, diminishing as $R_{\text {eq }}$ moves away from either $R_{\text {in }}$ or $R_{\text {out }}$. As expected, using the regular diffusion at operating temperature (i.e $D_{\mathrm{L}}=D$ ) is inadequate: FWHM is nearly double the true value (Figure 2a). Employing the Einstein equation with a higher $T$ from two-temperature treatment [48] narrows the peak somewhat. Finally, a yet higher $T_{\mathrm{L}}$ and $D_{\mathrm{L}}$ by eqs 7,8 augment the diffusion further, bringing the calculated FWHM within $150 \%$ of the measurement.

Now we turn the ion current on, while modeling the diffusion via "exact" eqs 7,8 . The peak profiles are graphed in Figure 2b, and the underlying trajectories are plotted in Figure $3 \mathrm{~b}$ and $\mathrm{d}$. The peak narrows as $I_{\text {in }}$ is raised to $180 \mathrm{pA}$, but remains unchanged at higher $I_{\mathrm{in}}$. The profile "freezes" at $I_{\text {in }} \geq 180 \mathrm{pA}$ because the current exceeding that value is rapidly reduced through Coulomb repulsion early in the simulation. This space-charge driven "explosion" is apparent in Figure $3 b$ and d. In particular (Figure 3d), ions are lost at both electrodes even when $R_{\mathrm{eq}}$ is much closer to $R_{\mathrm{in}}$ than to $R_{\text {out }}$ (or vice versa).
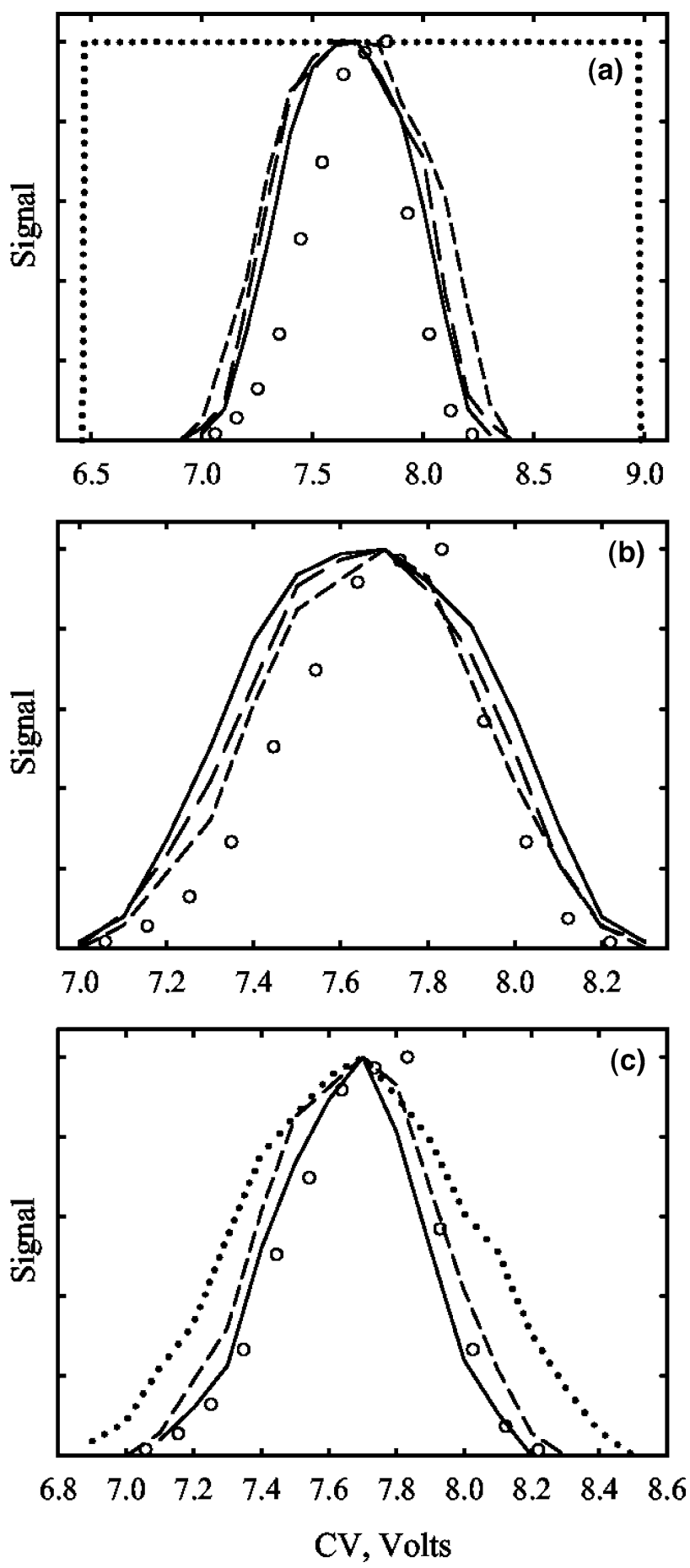

Figure 2. Profile of the (Leucine $-\mathrm{H})^{-}$in FAIMS CV spectrum acquired in air at DV $=-3.3 \mathrm{kV}$, experiment [28] (circles) and calculations (lines). (a) Shows the results using various models for diffusion (dotted rectangle-no diffusion, short dash line-thermal diffusion with $D_{\mathrm{L}}=D$, long dash-Einstein equation with a two-T treatment, solid-the "exact" treatment of eqs 7, 8). No Coulomb repulsion is included $\left(I_{\text {in }} \rightarrow 0\right)$. (b) Shows the influence of initial ion current: solid line for $I_{\text {in }} \rightarrow 0$ \{same as the solid line in (a)\}, long dash for $I_{\text {in }}=45 \mathrm{pA}$, and short dash for $I_{\text {in }}=180 \mathrm{pA}$. The diffusion is treated "exactly". (c) Shows the effect of varying the ion residence time: dotted line for $t_{\text {res }}=0.1 \mathrm{~s}$, short dash for $t_{\text {res }}=0.2 \mathrm{~s}$ \{same as the short dash in (b) \}, and solid for $t_{\text {res }}=0.4 \mathrm{~s}$. The diffusion is treated "exactly" and the ion current is saturated. 


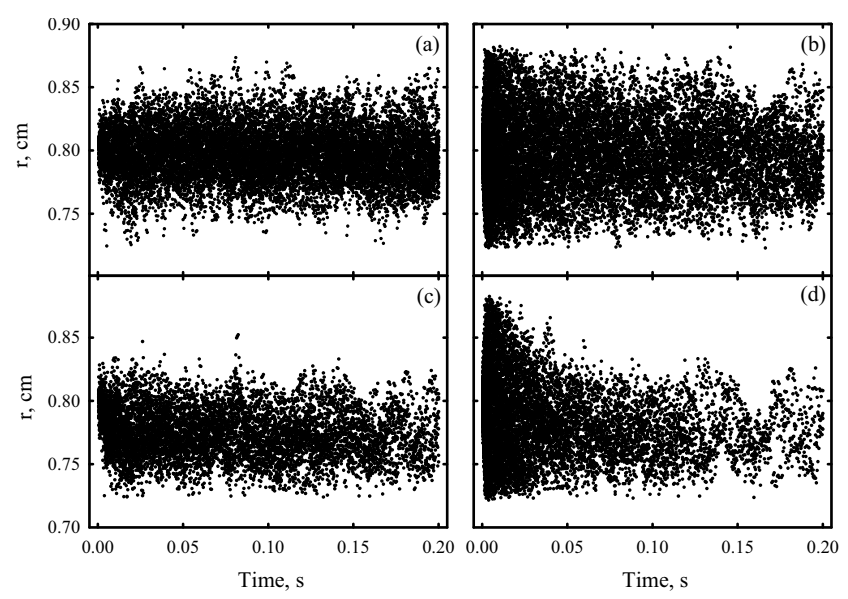

Figure 3. Evolution of ion populations in the simulations of Figure 2a, "exact" treatment (left column) and Figure $2 b, I_{\text {in }}=600$ pA (right column). For clarity, we show a representative subset of ions after each 100th FAIMS cycle $(0.48 \mathrm{~ms})$, i.e., oscillations within cycles are removed. The top panel is for $\mathrm{CV}=7.7 \mathrm{~V}$ (the apex of CV spectral peaks, $R_{\text {eq }}=0.80 \mathrm{~cm}$ ), the bottom is for $\mathrm{CV}=8.1 \mathrm{~V}$ (side of the peaks, $R_{\mathrm{eq}}=0.77 \mathrm{~cm}$ ). Ions are lost at both electrodes in (d), but only at the internal electrode in (c).

In contrast, the diffusional losses at $I_{\text {in }} \rightarrow 0$ occur only at the electrode nearest to $R_{\text {eq }}$ (Figure 3c). The attenuation of ion current through FAIMS is quantified in Figure 4. At low $I_{\text {in }}$ (e.g., $45 \mathrm{pA}$ ), ions are lost primarily by diffusion at a near-constant speed. At higher $I_{\mathrm{in}}$ the curves start exhibiting an initial rapid ion loss due to Coulomb repulsion followed by slower depletion via diffusion. Once the FAIMS charge capacity is filled at $I_{\text {in }} \sim 180 \mathrm{pA}$, within a few percent of the residence time the excess current is shed and all properties of transmitted ion ensemble (including the CV peak profile) become independent of $I_{\mathrm{in}}$.

The peak computed for $I_{\text {in }} \geq 180$ pA substantially

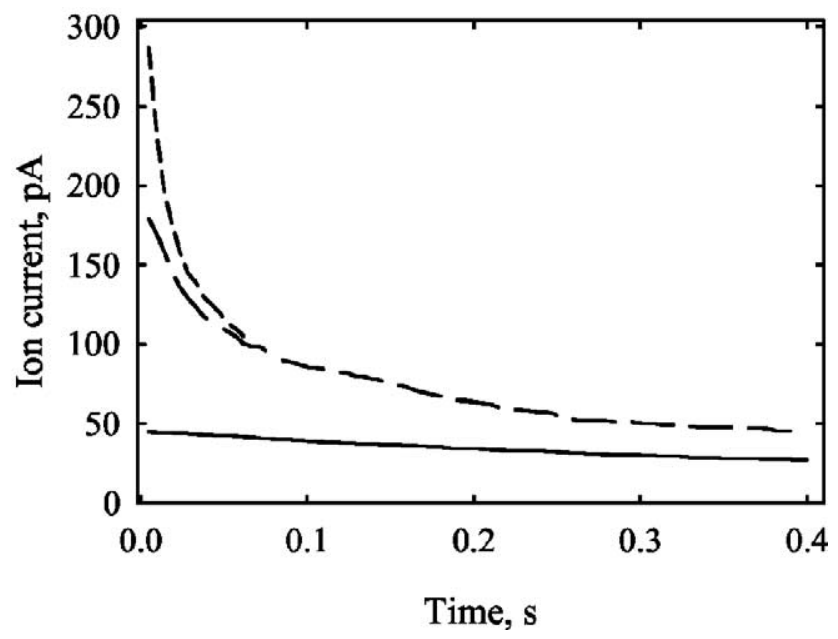

Figure 4. Calculated attenuation of (Leucine $-\mathrm{H})^{-}$ion current (in air) along the FAIMS gap (for DV $=-3.3 \mathrm{kV}$ and $\mathrm{CV}=7.7$ $\mathrm{V}$-peak apex). The lines are: solid for $I_{\text {in }}=45 \mathrm{pA}$, long dash for $I_{\text {in }}$ $=180 \mathrm{pA}$, and short dash for $I_{\mathrm{in}}=300 \mathrm{pA}$. These simulations assumed $t_{\text {res }}=0.4 \mathrm{~s}$ and $L=18 \mathrm{~cm}$, to keep the same surface ion density as for $t_{\text {res }}=0.2 \mathrm{~s}$ and $L=9 \mathrm{~cm}$ used in other graphs.

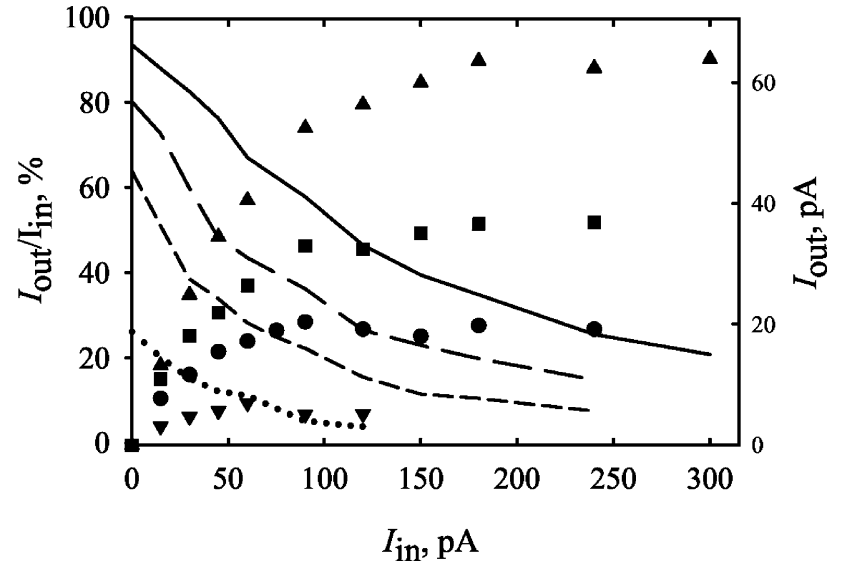

Figure 5. Transmission through FAIMS (at optimum CV) computed for (Leucine $-\mathrm{H})^{-}$in air. Symbols are transmitted currents (right axis) and lines are transmission coefficients (left axis): Triangles up/solid line for $\mathrm{DV}=-3.3 \mathrm{kV}(\mathrm{CV}=7.7 \mathrm{~V})$, squares/ long dash for $\mathrm{DV}=-2.7 \mathrm{kV}(\mathrm{CV}=4.53 \mathrm{~V})$, circles/short dash for $\mathrm{DV}=-2.3 \mathrm{kV}(\mathrm{CV}=2.93 \mathrm{~V})$, and triangles down/dotted for DV $=-1.7 \mathrm{kV}(\mathrm{CV}=1.26 \mathrm{~V})$. For all DV, the saturation of $I_{\text {out }}$ has been verified up to $I_{\text {in }}=1 \mathrm{nA}$.

reproduces the measurement, but is still broader by $10-20 \%$ (Figure $2 \mathrm{~b}$ ). This residual discrepancy may be due to the experimental residence time exceeding $0.2 \mathrm{~s}$ assumed in the calculations of Figure $2 a$ and $b$. In simulation, features always sharpen (i.e., the separation quality improves) as $t_{\text {res }}$ increases, because the ions with $R_{\text {eq }}$ near electrodes that make up the edges of peaks in CV spectra are disproportionately depleted by diffusion and Coulomb repulsion as time goes on. For example, Figure 2c shows a significant narrowing of (Leucine $\mathrm{H})^{-}$peaks between $t_{\text {res }}=0.1 \mathrm{~s}$ and $0.4 \mathrm{~s}$. The calculated and measured peaks match perfectly for $t_{\text {res }}=0.4 \mathrm{~s}$, a value within the experimental range [35]. Other possible reasons for the slight broadening of simulated peaks are discussed in conclusions.

To probe the current-limiting function of FAIMS further, we compute $I_{\text {out }}$ (at optimum CV values) as a function of $I_{\text {in }}$ at different DV (Figure 5). As one would expect, $I_{\text {out }}$ is roughly proportional to $I_{\text {in }}$ at low currents. As $I_{\text {in }}$ increases, the transmission efficiency goes down and $I_{\text {out }}$ reaches the saturation level, $I_{\text {sat }}$. This may equivalently be expressed in terms of charge capacity equal to $\int_{0}^{\text {tres }} I(t) d t \sim I_{\text {sat }} \times t_{\text {ress }}$, e.g., $1.2 \times 10^{-11} \mathrm{Cl}$ when $I_{\text {sat }}=60 \mathrm{pA}$ and $t_{\text {res }}=0.2 \mathrm{~s}$. Similar charge-capacity limitations exist for other ion guides and filters, including quadrupoles and higher-order multipoles, stackedring ion guides, and funnels [55]. At DV $=0$ (not plotted due to scale), the current increases from zero (with $I_{\text {out }} / I_{\text {in }}=8 \%$ ) to $I_{\text {sat }}=1.5 \mathrm{pA}$ reached at $I_{\text {in }} \sim 30$ pA. The transmission efficiencies approach unity only at highest DV values, even for vanishing $I_{\text {in }}$. The efficiencies at saturation decrease at lower DV: from $\sim 35 \%$ at $\mathrm{DV}=-3.3 \mathrm{kV}$ to $\sim 5 \%$ at $\mathrm{DV}=0$.

The $I_{\text {sat }}$ levels rise with increasing DV (Figure 5). A dramatic increase of the sensitivity of cylindrical FAIMS at higher DV is well-established, and qualitatively ex- 
plained as a manifestation of ion focusing [22, 28]. This phenomenon can now be quantified and investigated for different FAIMS mechanical and operational parameters. The relative values of $I_{\text {sat }}$ computed for a particular analyte at various DV should match measured signal intensities to the extent that four conditions hold. First, the incoming current of analyte ions must exceed $I_{\text {in }}$ at which $I_{\text {out }}$ saturates, so that $I_{\text {out }}$ would depend on the FAIMS transmission only. Whether this was the case for published work is difficult to ascertain. While the ESI source current was estimated [26, 32, 37, 38] as 30-60 nA, its fraction for the analyte of interest, the extent of desolvation prior to FAIMS injection, and the transmission efficiency of ESI/FAIMS pinhole interface are unknown. Second, no other ions may be injected concurrently with the ion of interest: those will initially fill a part of the charge capacity, even if subsequently lost to the FAIMS filtering action. This includes solvated analyte ions and other isomers (conformations) of the analyte. Generally, this condition is not met: MS commonly reveals multiple ions when FAIMS is disabled [28]. Their importance as competitors for the charge capacity depends on the speed of their elimination by FAIMS action versus the speed of analyte loss by Coulomb expansion, which would be significantly controlled by the $\mathrm{CV}$ difference between them and the analyte. The investigation of this issue is beyond the scope of this contribution. Third, analyte ions must not dissociate or isomerize inside FAIMS, else they could also be lost and not reach the MS stage. This should hold for most simple small ions that exhibit a single peak in CV spectra (trivially including all monatomic ions), but may be untrue for floppy macromolecular ions like proteins. For FAIMS/MS data, there is an additional condition of linear MS quantitation, i.e., the signal registered by the MS detector must scale with $I_{\text {out }}$. This should largely be satisfied by a quadrupole MS stage, such as PE Sciex API 300 used in much of FAIMS/MS work [22-26].

The departures from the above stipulations would affect the dependence of FAIMS/MS sensitivity on DV as follows. If the full charge capacity is not utilized above certain DV (violating the condition 1), $I_{\text {out }}$ would fall below $I_{\text {sat }}$ A nonlinear MS quantitation (violating the condition 4), whether caused by charge-capacity limitations of the MS stage or a detector saturation, would likewise flatten the slope of FAIMS/MS intensity as a function of DV. A failure to meet the condition 3 would reduce the ion count by the total fractional yield of isomerization and/or dissociation in FAIMS. As those processes would be promoted by stronger field heating, again the intensities at higher DV would be diminished disproportionately. Violating the condition 2 would reduce the signal by a factor equal to the analyte fraction in original ion mixture, with no obvious impact on the relative intensity versus DV. So any deviations from conditions (1-4) are expected to render the measured curve of (FAIMS/MS sensitivity versus DV) less steep than the ideal one derived from simulations.

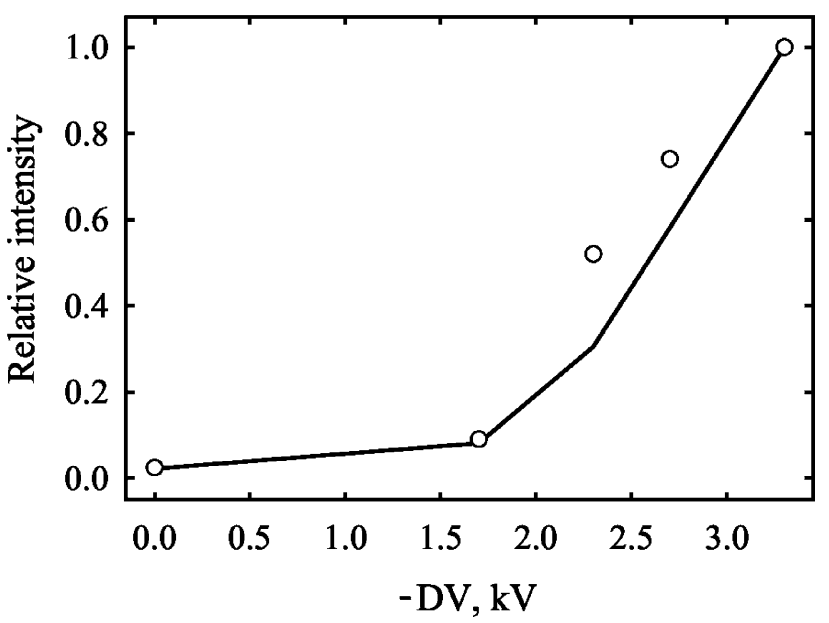

Figure 6. FAIMS sensitivity as a function of DV for (Leucine $\mathrm{H})^{-}$in air, calculation (at optimum CV values) (solid line) and FAIMS/MS measurements [28] (circles). In experiment, the features for (Leucine $-\mathrm{H})^{-}$fully or partially overlap with those for (Isoleucine $-\mathrm{H})^{-}$, which complicates quantitation. All intensities are normalized to 1.0 at the maximum $\mathrm{DV}=-3.3 \mathrm{kV}$.

The FAIMS transmission calculated for (Leucine $\mathrm{H})^{-}$and measured [28] FAIMS/MS sensitivity are plotted in Figure 6. Overall, between DV $=0$ (FAIMS disabled) and maximum DV of $-3.3 \mathrm{kV}$, the signal grew by a factor of $\sim 40-45$ in experiment versus 45 in calculations. The agreement is clearly good, especially considering the limitations of comparison outlined above and the analyte contamination by isoleucine [28]. The mobility properties for (Isoleucine $-\mathrm{H})^{-}$[30] are close to those for (Leucine $-\mathrm{H})^{-}$(Table 1) and our calculations for peak width and transmission efficiency yield similar results. Those are in an equally good agreement with the measurements [28].

\section{Calculations and Experiment for Other Amino Acids}

Within a class of compounds, the $K_{\mathrm{h}} / K(0)$ ratio tends to increase with decreasing ion size. Hence, when amino acid ions are ranked by values of $a$ in eq 1, leucine and isoleucine ( $m=131 \mathrm{Da})$ end in the middle and thus are "average" amino acids from the perspective of FAIMS response [30]. At the extremes are tryptophan-the largest amino acid $(m=204 \mathrm{Da})$ with weakest $K(E)$ dependence and glycine - the smallest amino acid $(m=$ $75 \mathrm{Da})$ with steepest dependence [30]. Transmission efficiencies computed for $\mathrm{H}^{+}$tryptophan and $\mathrm{H}^{+}$glycine (both belong to A-type and pass in P1 mode) are compared with those for (Leucine $-\mathrm{H})^{-}$in Figure 7a. As expected, ions with higher $K_{\mathrm{h}} / K(0)$ experience a stronger focusing in cylindrical FAIMS and thus allow higher currents: $I_{\text {sat }}$ ascend from $\sim 8 \mathrm{pA}$ for tryptophan to $\sim 64 \mathrm{pA}$ for leucine to $\sim 115 \mathrm{pA}$ for glycine. The transmission efficiencies at any $I_{\text {in }}$ increase likewise, e.g., those at $I_{\text {sat }}$ go up from $\sim 15 \%$ for tryptophan tox 

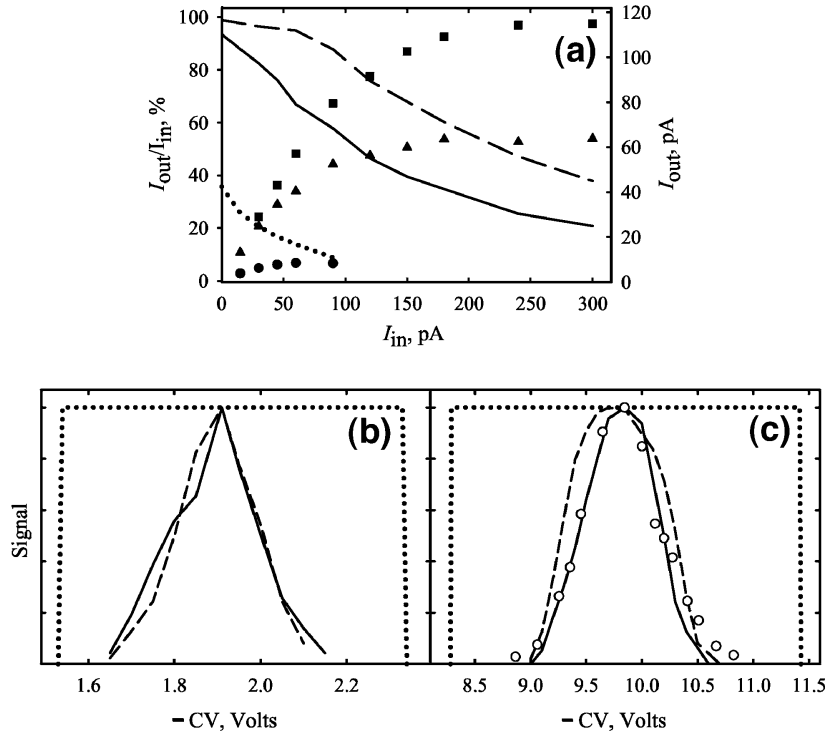

Figure 7. (a) Same as Figure 5 for three amino acid ions in air (at $\mathrm{DV}= \pm 3.3 \mathrm{kV}$ ). Squares/dashed line is for $\mathrm{H}^{+}$glycine, triangles/ solid line for (Leucine $-\mathrm{H})^{-}$, and circles/dotted line for $\mathrm{H}^{+}$tryptophan. (b and c) Profiles of $\mathrm{H}^{+}$tryptophan $(\mathbf{b})$ and $\mathrm{H}^{+}$glycine (c) peaks in $\mathrm{CV}$ spectra in $\mathrm{N}_{2}$ gas $(\mathrm{DV}=3.0 \mathrm{kV})$, calculations (lines) and FAIMS/MS measurements (circles in (c) from [29]). Solid lines are for current-saturated conditions, dashed lines are for $I_{\text {in }} \rightarrow 0$, and dotted rectangles are for the case of no diffusion.

$\sim 35 \%$ for leucine to $\sim 50 \%$ for glycine. All these trends as a function of $K_{\mathrm{h}} / K(0)$ at a fixed DV are quite similar to those for a single species as a function of DV (Figure $5)$. This happens because focusing is administered by $K(E) \times E$ product in eq 5 .

A drastic dependence of $I_{\text {sat }}$ on $K_{\mathrm{h}} / K(0)$ affects the modeled peak profile. When $K(E)$ dependence is weak and ion current is low even at saturation, Coulomb repulsion has essentially no effect on the peak shape. This is the case for $\mathrm{H}^{+}$tryptophan (Figure $7 \mathrm{~b}$ ), where the peaks for $I_{\text {in }} \rightarrow 0$ and at saturation are indistinguishable within the computational accuracy. For $\mathrm{H}^{+}$glycine (Figure 7c), FWHM computed at maximum current is $\sim 0.7$ of that at $I_{\text {in }} \rightarrow 0$ (the value for Leucine was $>0.8$ ). The peak profile calculated at saturation is in an excellent agreement with FAIMS/MS measurements [29] (Figure 7c). This is particularly significant considering that strong $K(E)$ dependence and high ion currents make $\mathrm{H}^{+}$glycine the toughest amino acid to model.

\section{Small Ions: The Case of Bisulfate Anion}

Other major FAIMS applications are in environmental analysis, for example drinking water quality control. Typical targets are small halogen-containing molecules that are common groundwater contaminants, such as haloacetic acids [37, 56] and (per)halogenates [24-26]. Bisulfate anion $\left(\mathrm{HSO}_{4}^{-}\right)$with ${ }^{34} \mathrm{~S}$ or ${ }^{18} \mathrm{O}$ natural isotopes ( $m=99 \mathrm{Da})$ is a ubiquitous mass interference in perchlorate assays, and mixture of $\mathrm{HSO}_{4}^{-}$and $\mathrm{ClO}_{4}^{-}$has
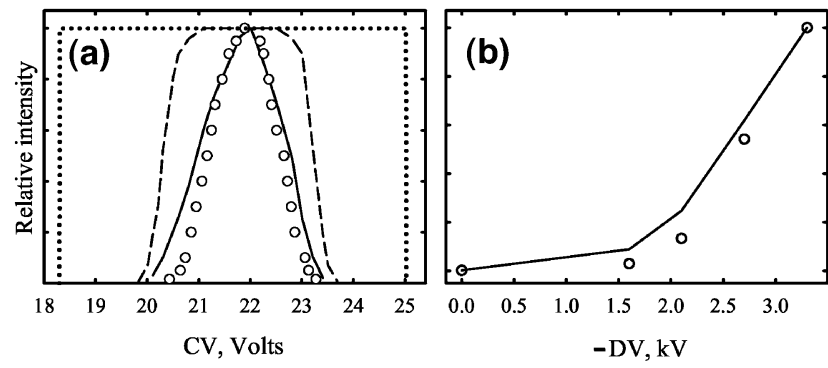

Figure 8. FAIMS/MS response of $\mathrm{HSO}_{4}^{-}$in air: The peak profile at $\mathrm{DV}=3.3 \mathrm{kV}(\mathbf{a})$ and relative sensitivity as a function of DV (normalized to 1.0 at maximum DV $=-3.3 \mathrm{kV})(\mathbf{b})$. Circles stand for measurements, lines are for calculations. In (a), solid line is for saturated ion current, dashed is for $I_{\text {in }} \rightarrow 0$, and dotted rectangle is computed ignoring diffusion.

been probed [25] by FAIMS/MS. Both are A-type ions and pass in $\mathrm{N} 1$ mode. The $K(E)$ dependences for small inorganic ions are normally stronger than that for any amino acid (Table 1), which results in higher CV s and greater ion currents. To test the simulation at those stringent conditions, we chose the case of $\mathrm{HSO}_{4}^{-}$. Modeled and measured [25] peaks in CV spectra are compared in Figure 8a. The FWHM calculated at saturation is about half that at $I_{\text {in }} \rightarrow 0$, because of powerful Coulomb repulsion at very high ion currents: $I_{\text {sat }} \sim 290$ $\mathrm{pA}$ is attained at $I_{\mathrm{in}} \sim 420 \mathrm{pA}$. The transmission efficiency of $70 \%$ is above $50 \%$ for $\mathrm{H}^{+}$glycine, confirming the trend for transmission to increase at higher $I_{\text {sat. }}$. This indicates a transmission controlled mostly by the balance between Coulomb repulsion and FAIMS focusing, with diffusion increasingly irrelevant. This regime presents the most severe test for the ability of simulation to handle space-charge phenomena. The peak FWHM evaluated at saturation exceeds the measurement [25] by $\sim 20 \%$, about as much as for (Leucine $-\mathrm{H})^{-}$. Relative $I_{\text {sat }}$ current as a function of DV is plotted in Figure $8 \mathrm{~b}$. The agreement with experiment [25] is good, particularly considering the presence of competing perchlorate ion and general limitations arising from FAIMS/MS coupling (see prior section).

With increasing DV, sensitivity improves more for smaller ions with stronger focusing than for larger ions. For example, between DV $=0$ and maximum DV of $-3.3 \mathrm{kV}$, measured signal grew $\sim 400$ times for $\mathrm{HSO}_{4}^{-}$ versus only 40-45 times for leucine/isoleucine anions. Modeling replicates this effect, yielding a factor of 360 for $\mathrm{HSO}_{4}^{-}$. The underlying physics is that smaller ions have higher mobilities and thus diffuse in gases faster, but also tend to have steeper $K(E)$ dependences and thus focus in cylindrical FAIMS stronger. Hence the transmission efficiencies for larger ions exceed those for smaller ones at low DV, e.g., at DV $=0$ (FAIMS disabled) $I_{\text {sat }}=0.8 \mathrm{pA}$ for $\mathrm{HSO}_{4}^{-}$but $I_{\mathrm{sat}}=1.4 \mathrm{pA}$ for (Leucine $-\mathrm{H})^{-}$. At high DV, the opposite is true as discussed above. 


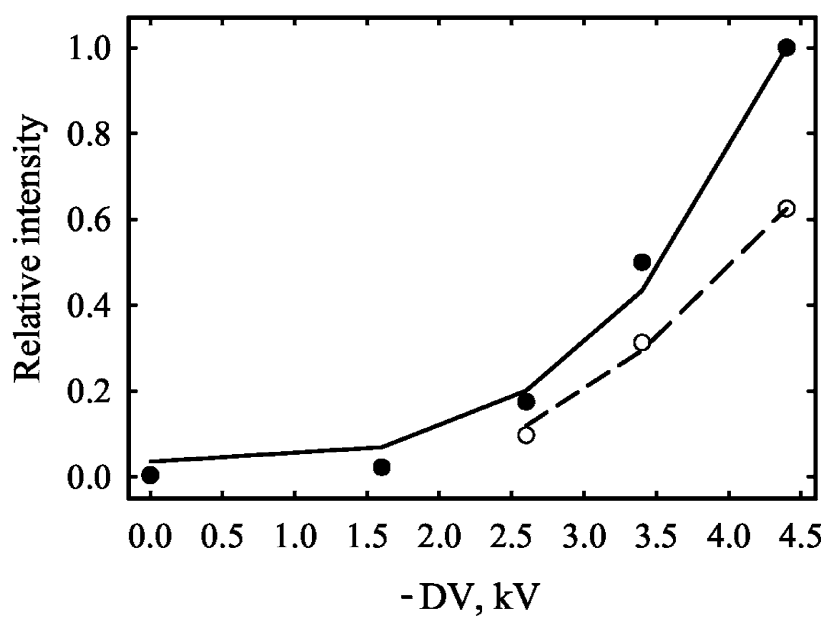

Figure 9. Same as Figure $8 \mathrm{~b}$ for protonated bovine ubiquitin (+12 charge state) in $\mathrm{N}_{2}$. Filled circles and solid lines stand for the major isomer, empty circles and dashed lines are for the minor isomer.

\section{Macromolecular Ions: The Bovine Ubiquitin Example}

At the other end of mass range from small ions lie macromolecular species such as proteins. Several proteins have been examined in FAIMS, with bovine ubiquitin (a common small protein of $8.6 \mathrm{kDa}$ mass) studied most thoroughly [40, 42, 57]. As most large ions, it belongs to C-type and passes FAIMS in P2 mode. Fast post-ionization separations of protein mixtures using FAIMS may find application in topdown proteomics $[58,59]$, offering the same throughput advantage over condensed-phase methods as when separating proteolytic digests in bottom-up approaches. FAIMS separations of proteins are also largely orthogonal to both IMS and MS dimensions $[40,42]$. Different protein conformations are also distinguished, which may allow one to access protein polymorphism in solution [40, 42, 57]. Since many human and animal diseases are associated with protein misfolding $[60,61]$, this would have major biomedical significance.

To test the simulation for proteins, we have modeled the FAIMS/MS intensity for Bovine Ubiquitin as a function of DV. This protein typically picks up 5-15 protons in ESI process [40,42]. Calculations for both major and minor isomers of $(+12)$ charge state match the measurements [40] nearly perfectly across the whole DV range, here extending to $4.4 \mathrm{kV}$ (Figure 9). In accordance with experiment [40], these simulations were run at $(w / 2 \pi)$ equal to $750 \mathrm{kHz}$ rather than 210 $\mathrm{kHz}$ for the data in Figures 2-8. An excellent agreement in Figure 9 supports the verity of simulation at different waveform frequencies, and at higher DV. This also demonstrates the ability of model to handle C-type ions, and very large ions that have relatively weak $K(E)$ dependences, low mobilities, and particularly low diffusion constants. Data [40] were acquired using a FAIMS with hemispherical end that is not incorporated into present simulations. The agreement in Figure 9 suggests that, to the first approximation, the model can be applied even to that geometry.

\section{Conclusions}

We have developed a realistic simulation describing the ion dynamics in field asymmetric waveform ion mobility spectrometry (FAIMS) analyzers. The model effectively handles both the planar parallel plate geometry and the common coaxial cylinder design with any curvature. The theoretical approach involves an ensemble of ions injected into FAIMS evolving over the full residence time under the influence of external electric field, random gas diffusion (including the high-field and anisotropic components), and mutual Coulomb repulsion. A number of statistical parameters characterizing that ensemble may be collected in the course of simulation, which allows modeling of various experimental observables. In particular, calculation of the ion transmission efficiencies across a range of compensation voltages yields the $\mathrm{CV}$ spectral profiles that characterize the resolution of FAIMS experiments. Evaluation of these efficiencies (at optimum CV) as a function of dispersion voltage and other parameters determines the FAIMS sensitivity that may also be compared with the measurements. The diffusion and Coulomb repulsion always spatially expand the ion packets, which decreases the resolving power of IMS and massspectrometric techniques in general. Contrarily, this broadening improves the FAIMS resolution by preferentially eliminating ions at the edges of $\mathrm{CV}$ spectral peaks that pass near the electrodes.

The results derived from this model have been matched with experimental findings on FAIMS/MS resolution and sensitivity. The assessment included several amino acids that are representative of organic and smaller biological ions of primary interest in bottom-up proteomics and metabolomics, a typical protein encountered in top-down proteomics, and a small inorganic ion-a common target of environmental analyses. This test set comprises both cations and anions, and ions of both A-type and C-type. Altogether, these ions pass FAIMS in P1, P2, and N1 modes - three modes out of four possible [21]. The instrumental sensitivities (relative values as a function of dispersion voltage) are reproduced well for all cases, including those involving high ion currents up to hundreds of picoAmperes. In particular, calculations replicate the observation that increasing DV improves the signal intensity for small ions more than for large ones. The ability to handle ion intensities over a dynamic range of at least three orders of magnitude portends well for the applicability of present treatment to various FAIMS designs and operational regimes. The agreement for $\mathrm{CV}$ spectra is excellent, with the computed peak widths matching measurements within $10-20 \%$.

Several reasons may lead the model to overestimate peak widths. First, simulations should use a realistic distribution of residence times rather than a fixed time. 
This distribution arises from (1) axial spread of ions by random diffusional motion superposed on the directed movement along the gap with the gas flow and (2) an uneven profile of flow velocity across the gap that is inevitable in a pipe of any geometry due to finite gas viscosity. The latter renders the resident time of an ion dependent on CV: species traveling near the gap median will pass FAIMS faster than those near the electrodes. Since the cumulative probability for an ion to be lost grows with time (Figure 4), this will narrow all CV spectral features. Second, imperfections of gas flow through the analytical gap and any electronic noise or "ripple" on the waveform would narrow the CV spectral features by depleting marginally stable ions at their edges. These ions may also be depleted by attraction to image charges created by the ion current on FAIMS electrodes. We are working to gauge the magnitude of these effects and account for them where warranted.

The present simulation environment is almost infinitely flexible, allowing the examination of FAIMS action for virtually any geometric and functional parameters, and arbitrary initial ion distributions. We expect it to be of utility in optimizing the FAIMS design and operation; these efforts are in progress.

\section{Acknowledgments}

The authors are grateful to Drs. R. Guevremont, R. Purves, S.E. Barlow, E.N. Nikolaev, and A. Tolmachev for many insightful discussions of FAIMS operation and its computational modeling. They thank the U.S. Department of Energy's Office of Biological and Environmental Research and the NIH National Center for Research Resources (RR18522) for supporting portions of this research. Pacific Northwest National Laboratory is operated by the Battelle Memorial Institute for the U.S. Department of Energy through contract DE-ACO6-76RLO1830.

\section{References}

1. Aebersold, R.; Mann, M. Nature 2003, 422, 198.

2. Griffin, J. L. Curr. Opin. Chem. Biol. 2003, 7, 648.

3. Shevchenko, A.; Wilm, M.; Vorm, O.; Mann, M. Anal. Chem. 1996, 68, 850 .

4. Shen, Y.; Zhao, R.; Berger, S. J.; Anderson, G. A.; Rodriguez, N.; Smith, R. D. Anal. Chem. 2002, 74, 4235.

5. Washburn, M. P.; Wolters, D.; Yates, J. R. Nature Biotechnol. 2001, 19, 242.

6. Shen, Y.; Xiang, F.; Veenstra, T. D.; Fung, E. N.; Smith, R. D. Anal. Chem. 1999, 71, 5348.

7. Wolters, D. A.; Washburn, M. P.; Yates, J. R. Anal. Chem. 2001, 73, 5683.

8. Hill, Jr.; H. H., Siems, W. F.; St. Louis, R. H.; McMinn, D. G. Anal. Chem. 1990, 62, A1201.

9. Eiceman, G. A. Crit. Rev. Anal. Chem. 1991, 22, 17.

10. von Helden, G.; Hsu, M. T.; Kemper, P. R.; Bowers, M. T. J. Chem. Phys. 1991, 95, 3835.

11. Dugourd, P.; Hudgins, R. R.; Clemmer, D. E.; Jarrold, M. F. Rev. Sci. Instrum. 1997, 68, 1122.

12. Fromherz, R.; Ganteför, G.; Shvartsburg, A. A. Phys. Rev. Lett. 2002, 89, 083001.

13. Guevremont, R.; Siu, K. W. M.; Wang, J. Y.; Ding, L. Anal. Chem. 1997, 69, 3959.
14. Bluhm, B. K.; Gillig, K. J.; Russell, D. H. Rev. Sci. Instrum. 2000, $71,4078$.

15. Srebalus Barnes, C. A.; Clemmer, D. E. Anal. Chem. 2001, 73, 424.

16. Hoaglund-Hyzer, C. S.; Lee, Y. J.; Counterman, A. E.; Clemmer, D. E. Anal. Chem. 2002, 74, 992.

17. Buryakov, I. A.; Krylov, E. V.; Nazarov, E. G.; Rasulev, U. K. Int. J. Mass Spectrom. Ion Processes 1998, 128, 143.

18. Krylov, E.; Nazarov, E. G.; Miller, R. A.; Tadjikov, B.; Eiceman, G. A. J. Phys. Chem. A 2002, 106, 5437.

19. Krylova, N.; Krylov, E.; Eiceman, G. A.; Stone, J. A. J. Phys. Chem. A 2003, 107, 3648.

20. Carnahan, B. L., Tarassov, A. S. U.S. Patent no. 5420424; 1995.

21. Purves, R. W.; Guevremont, R.; Day, S.; Pipich, C. W.; Matyjaszczyk, M. S. Rev. Sci. Instrum. 1998, 69, 4094.

22. Guevremont, R.; Purves, R. W. Rev. Sci. Instrum. 1999, 70, 1370.

23. Viehland, L. A.; Guevremont, R.; Purves, R. W.; Barnett, D. A. Int. J. Mass Spectrom. 2000, 197, 123.

24. Barnett, D. A.; Guevremont, R.; Purves, R. W. Appl. Spectrosc. 1999, 53, 1367.

25. Handy, R.; Barnett, D. A.; Purves, R. W.; Horlick, G.; Guevremont, R. J. Anal. At. Spectrom. 2000, 15, 907.

26. Ells, B.; Barnett, D. A.; Purves, R. W.; Guevremont, R. J. Environ. Monit. 2000, 2, 393.

27. Barnett, D. A.; Purves, R. W.; Guevremont, R. Nucl. Instrum. Methods A 2000, 450, 179.

28. Barnett, D. A.; Ells, B.; Guevremont, R.; Purves, R. W. J. Am. Soc. Mass Spectrom. 1999, 10, 1279.

29. Purves, R. W.; Guevremont, R. Anal. Chem. 1999, 71, 2346.

30. Guevremont, R.; Barnett, D. A.; Purves, R. W.; Viehland, L. A. J. Chem. Phys. 2001, 114, 10270.

31. Guevremont, R.; Purves, R. W. J. Am. Soc. Mass Spectrom. 1999, 10, 492.

32. Barnett, D. A.; Purves, R. W.; Ells, B.; Guevremont, R. J. Mass Spectrom. 2000, 35, 976.

33. Purves, R. W.; Barnett, D. A.; Ells, B.; Guevremont, R. Rapid. Commun. Mass Spectrom. 2001, 15, 1453.

34. Guevremont, R.; Ding, L.; Ells, B.; Barnett, D. A.; Purves, R. W. J. Am. Soc. Mass Spectrom. 2001, 12, 1320.

35. Guevremont, R.; Barnett, D. A.; Purves, R. W.; Vandermey, J. Anal. Chem. 2000, 72, 4577.

36. Barnett, D. A.; Ells, B.; Guevremont, R.; Purves, R. W. J. Am. Soc. Mass Spectrom. 2002, 13, 1282.

37. Ells, B.; Barnett, D. A.; Froese, K.; Purves, R. W.; Hrudey, S.; Guevremont, R. Anal. Chem. 1999, 71, 4747.

38. McCooeye, M.; Ding, L.; Gardner, G. J.; Fraser, C. A.; Lam, J.; Sturgeon, R. E; Mester, Z. Anal. Chem. 2003, 75, 2538.

39. Gabryelski, W.; Wu, F.; Froese, K. L. Anal. Chem. 2003, 75, 2478.

40. Purves, R. W.; Barnett, D. A.; Ells, B.; Guevremont, R. J. Am. Soc. Mass Spectrom. 2001, 12, 894.

41. Cui, M.; Ding, L.; Mester, Z. Anal. Chem. 2003, 75, 5847.

42. Purves, R. W.; Barnett, D. A.; Ells, B.; Guevremont, R. J. Am. Soc. Mass Spectrom. 2000, 11, 738.

43. Jarrold, M. F. J. Phys. Chem. 1995, 99, 11.

44. Shvartsburg, A. A.; Jarrold, M. F. Phys. Rev. A 1999, 60, 1235.

45. Shvartsburg, A. A.; Hudgins, R. R.; Dugourd, P.; Jarrold, M. F. J. Phys. Chem. A 1997, 101, 1684.

46. James, D. R.; Graham, E.; Akridge, G. R.; Gatland, I. R.; McDaniel, E. W. J. Chem. Phys. 1975, 62, 1702.

47. Viehland, L. A.; Mason, E. A. J. Chem. Phys. 1975, 63, 2913.

48. Shvartsburg, A. A.; Schatz, G. C.; Jarrold, M. F. J. Chem. Phys. 1998, 108, 2416.

49. Shelimov, K. B.; Jarrold, M. F. J. Am. Chem. Soc. 1997, 119, 2987.

50. Chaurand, P.; Stoeckli, M.; Caprioli, R. M. Anal. Chem. 1999, 71, 5263.

51. Whealton, J. H.; Mason, E. A. Phys. Rev. A 1972, 6, 1939. 
52. Whealton, J. H.; Mason, E. A.; Robson, R. E. Phys. Rev. A 1974, 9, 1017.

53. Milloy, H. B.; Robson, R. E. J. Phys. B 1973, 6, 1139.

54. Wu, J. Y.; Kao, H. J.; Li, S. C.; Stevens, R.; Hillman, S.; Millington, D.; Chen, Y. T. J. Clin. Invest. 2004, 113, 434.

55. Tolmachev, A. V.; Udseth, H. R.; Smith, R. D. Anal. Chem. 2000, 72, 970.

56. Ells, B.; Barnett, D. A.; Purves, R. W.; Guevremont, R. Anal. Chem. 2000, 72, 4555.

57. Purves, R. W.; Barnett, D. A.; Guevremont, R. Int. J. Mass Spectrom. 2000, 197, 163.
58. Reid, G. E.; McLuckey, S. A. J. Mass Spectrom. 2002, 37, 63.

59. Taylor, G. K.; Kim, Y. B; Forbes, A. J.; Meng, F.; McCarthy, R.; Kelleher, N. L. Anal. Chem. 2003, 75, 4081.

60. Soto, C. FEBS Lett. 2001, 498, 204.

61. Valentine, J. S.; Hart, P. J. Proc. Natl. Acad. Sci. U.S.A. 2003, 100, 3617.

62. Barnett, D. A.; Ells, B.; Guevremont, R.; Purves, R. W.; Viehland, L. A. J. Am. Soc. Mass Spectrom. 2000, 11, 1125.

63. Li, J. W.; Taraszka, J. A.; Counterman, A. E.; Clemmer D. E. Int. J. Mass Spectrom. 1999, 187, 37. 